\title{
Clinical Efficacy of Allergen-Specific Immunotherapy from Patient and Physician Perspectives
}

\author{
Yoo Seob Shin ${ }^{1 *}$, Jae-Woo Jung ${ }^{2 *}$, Jung-Won Park ${ }^{3}$, Jeong-Hee Choi ${ }^{4}$, Jae-Woo Kwon ${ }^{5}$, Sooyoung Lee ${ }^{6}$, \\ Ji Hye Kim ${ }^{7}$, Sang Min Lee ${ }^{8}$, Young Min Ahn ${ }^{9}$, and Man Yong Han ${ }^{10}$ on behalf of the Allergen and \\ Immunotherapy Work Group of the Korean Academy of Asthma, Allergy and Clinical Immunology (KAAACI) \\ ${ }^{1}$ Department of Allergy and Clinical Immunology, Ajou University School of Medicine, Suwon; \\ ${ }^{2}$ Department of Internal Medicine, Chung-Ang University College of Medicine, Seoul; \\ ${ }^{3}$ Department of Internal Medicine, Institute of Allergy, Yonsei University College of Medicine, Seoul; \\ ${ }^{4}$ Department of Pulmonology and Allergy, Hallym University College of Medicine, Chuncheon; \\ ${ }^{5}$ Department of Internal Medicine, Kangwon National University School of Medicine, Chuncheon; \\ ${ }^{6}$ Department of Pediatrics, Ajou University School of Medicine, Suwon; \\ ${ }^{7}$ Division of Pulmonology and Allergy, Konyang University College of Medicine, Daejeon; \\ ${ }^{8}$ Division of Pulmonology and Allergy, Department of Internal Medicine, Gachon University Gil Medical Center, Incheon; \\ ${ }^{9}$ Department of Pediatrics, Eulji General Hospital, Eulji University School of Medicine, Seoul; \\ ${ }^{10}$ Department of Pediatrics, CHA Bundang Medical Center, CHA University School of Medicine, Seongnam, Korea.
}

Purpose: Allergen-specific immunotherapy (AIT) is the only curative treatment for allergic diseases, but a few allergic patients receive AIT. In this multicenter cross-sectional study, we aimed to explore patient and physician perspectives on AIT through a questionnaire survey.

Materials and Methods: Allergic patients who received subcutaneous immunotherapy for at least 1 year were asked to answer a questionnaire developed by an expert panel of allergen and immunotherapy workgroup in Korea.

Results: A total of 267 patients (adults, 60.3\%) with allergic rhinitis (91.4\%), asthma (42.7\%), or atopic dermatitis (20.2\%) from referred hospitals completed the survey. Among patients and physicians, respectively, the overall rates of satisfaction with AIT for allergic rhinitis were $86.4 \%$ and $83.3 \%$ (kappa agreement $=0.234, p<0.001$ ), and those for asthma were $85.3 \%$ and $72.9 \%$ (kappa agreement $=0.373, p<0.001$ ). Moreover, pediatric asthmatic patients reported a significantly higher satisfaction rate than adult asthmatic patients after AIT ( $p=0.040)$. Symptom severity $(p<0.001$, respectively $)$ and drug use for allergic rhinitis and asthma decreased after AIT. However, there was no significant difference in satisfaction rates between children and adults in allergic rhinitis $(p=0.736)$. Interestingly, $35.7 \%$ and $35 \%$ of allergic rhinitis and asthma patients, respectively, reported experiencing improvement in their symptoms within 6 months of starting AIT.

Conclusion: In this study evaluating the perspectives of patients and physicians on AIT, the majority of patients were satisfied with the efficacy and safety of AIT, but not its cost. AIT should be recommended for AR and allergic patients.

Key Words: Allergen immunotherapy, patient satisfaction, surveys and questionnaire

Received: January 23, 2019 Revised: February 19, 2019 Accepted: March 13, 2019

Co-corresponding authors: Man Yong Han, MD, Department of Pediatrics, CHA Bundang Medical Center, CHA University School of Medicine, 59 Yatap-ro, Bundang-gu, Seongnam 13488, Korea.

Tel: 82-31-780-6262, Fax: 82-31-780-5239, E-mail: drmesh@gmail.com and

Young Min Ahn, MD, PhD, Department of Pediatrics, Eulji General Hospital, Eulji University School of Medicine, 68 Hangeulbiseok-ro, Nowon-gu, Seoul 01830, Korea.

Tel: 82-2-970-8228, Fax: 82-2-976-5441, E-mail: aym3216@eulji.ac.kr

*Yoo Seob Shin and Jae-Woo Jung contributed equally to this work.

-The authors have no potential conflicts of interest to disclose.

(C) Copyright: Yonsei University College of Medicine 2019

This is an Open Access article distributed under the terms of the Creative Commons Attribution Non-Commercial License (https://creativecommons.org/licenses/by-nc/4.0) which permits unrestricted non-commercial use, distribution, and reproduction in any medium, provided the original work is properly cited. 


\section{INTRODUCTION}

Since Leonard Noon and John Freeman reported the effect of prophylactic inoculation of grass pollen in hay fever patients in 1911, allergen-specific immunotherapy (AIT) has been used to treat allergic diseases in clinical practice for more than 100 years. ${ }^{1}$ It is well known that AIT can reduce symptoms and medication scores, ${ }^{2,3}$ alter the natural course of allergic disease $^{4,5}$ and prevent disease progression from allergic rhinitis to asthma ${ }^{6,7}$ as well as new sensitization. ${ }^{8,9}$ However, relatively long treatment duration, high cost, and frequent hyperreactivity reactions, including anaphylaxis, can be hurdles to choose AIT.

Although AIT is a well-established, effective therapeutic modality for treating allergic disease, the use of AIT is not widely used in most countries. A minor portion (2-9\%) of allergic patients in the United States have been treated with AIT according to previous reports, ${ }^{10,11}$ and only $36.2 \%$ of patients recommended AIT by their doctors doctors eventually underwent AIT. ${ }^{12}$ This seems to suggest a lack of education and understanding of AIT among both patients and doctors. Demoly, et al. ${ }^{13}$ reported that about $50 \%$ of patients recognized AIT as a therapeutic option, of whom $14 \%$ had negative opinion thereof. However, once AIT is started, approximately $60 \%$ of patients are satisfied with it. Among doctors, less than 50\% of general practitioners were aware of the exact placement of AIT, and almost all of them wanted detail information about AIT in Italy. ${ }^{14,15}$ Several aspects of AIT in controlled studies have been extensively investigated, including clinical efficacy, ${ }^{16-18}$ tolerability, ${ }^{19}$ and effects on health-related quality of life (QoL); ${ }^{20-22}$ however, a few studies addressing patient and physician perspectives on AIT have been published, especially in Korea.

Accordingly, this multicenter cross-sectional study aimed to explore patient and physician satisfaction with the efficacy, safety, and economic parameters of AIT through a questionnaire survey: this survey addressed symptom changes, medication use, economic aspects, side effects, and satisfaction rate among allergic patients, as well as changes in disease severity based on international guidelines.

\section{MATERIALS AND METHODS}

\section{Patients}

A total of 273 patients with allergic rhinitis, allergic conjunctivitis, allergic asthma, and/or atopic dermatitis were recruited from 10 secondary or tertiary hospitals around Korea between April 2017 and July 2018 in this study. Inclusion criteria were patients who gave written informed consent and received AIT to house dust mites, tree/grass/weed pollen, animal dander, or fungi for at least 1 year, regardless of AIT methods and manufacturing companies. Of the 273 patients, only six re- ceived sublingual immunotherapy (SLIT) for their allergic disease and were excluded from the study. The study protocol was approved by the institutional review boards of each hospital (AJIRB-MED-SUR-17-159, Ajou University Hospital).

\section{Survey}

The questionnaire was developed by an expert panel from the Korean Academy of Asthma, Allergy and Clinical Immunology (KAAACI) on the basis of experience and literature review. After a literature search for perspectives of AIT, workgroup members made a draft of this study. For each question, responses and comments from the workgroup members were collected and synthesized into a draft manuscript that was recirculated until consensus was reached. The questionnaire consisted of 15 items for patients and 10 items for physicians. The patient questionnaire included demographics, perception, expectation, side effects, and satisfaction on a 5-point Likert scale (much improved/improved/no difference/aggravated/much aggravated; excellent/good/fair/poor/very poor; very favorable/favorable/neutral/unfavorable/very unfavorable). The physician questionnaire included allergic status, AIT products, and changes in disease severity based on international guidelines. The questionnaire items for patients and physicians are presented in the Supplementary Materials (only online).

\section{Statistical analysis}

All analyses were carried out using SPSS 22.0 (IBM Corp., Armonk, NY, USA). Descriptive statistics were used to analyze demographic and clinical data obtained from the patients' and physicians' responses to the questionnaire. Continuous variables are expressed as means $\pm \mathrm{SD}$ and categorical variables are expressed as percentage. The clinical data were compared among age groups using the chi-square test or McNemar's test. All $p$ values $<0.05$ were considered statistically significant.

\section{RESULTS}

\section{General characteristics}

A total of 267 patients who received subcutaneous immunotherapy were analyzed in this study. Enrolled patients consisted of $161(60.3 \%)$ patients treated at the Department of Internal Medicine and 106 (39.7\%) treated at the Department of Pediatrics. The mean age was $25.6 \pm 15.4$ years, and $133(49.8 \%)$ were male. AIT was most frequently prescribed to patients in their 10s; it was rarely prescribed to patients in their $60 \mathrm{~s}$. In addition, $79.0 \%$ of patients had a family history of allergic disease, and $10.2 \%$ of patients had a previous AIT history. Most patients (69.6\%) had heard about AIT by general hospital physicians; however, only $5.9 \%$ of allergic patients had heard about AIT by general practitioners. 
Allergic rhinitis (91.4\%) was the most common disease to be treated by AIT, followed by asthma (42.7\%) and atopic dermatitis $(20.2 \%)$. Their mean duration of AIT was $27.3 \pm 15.0$ months. Moreover, $91.6 \%$ of patients reported that they had good adherence to AIT, and these data were correlated with that of the doctors' views (kappa agreement $=0.356, p<0.001$ ). Detailed data on the patient characteristics and immunotherapy-related factors are shown in Table 1.

\section{Changes in symptoms and disease severity after AIT}

After more than 1 year AIT of treatment for asthma, most patients reported a better than 'improved' state in their daytime symptoms (91.0\%), nighttime symptoms (92.8\%), short-acting beta-agonist use (82.4\%), asthma attack (87.2\%), and QoL (90.5\%) (Fig. 1A). Physician indicated in the questionnaire on allergy symptoms and medication use that disease severity

Table 1. Demographic- and Immunotherapy-Related Characteristics of Patients in This Study

\begin{tabular}{|c|c|}
\hline Variables & $\mathrm{n}=267(\%)$ \\
\hline Age (yr) & $25.6 \pm 15.4(1-69)$ \\
\hline $1-9$ & 35 \\
\hline $10-19$ & 82 \\
\hline $20-29$ & 45 \\
\hline $30-39$ & 51 \\
\hline $40-49$ & 30 \\
\hline $50-59$ & 20 \\
\hline $60-69$ & 3 \\
\hline Sex, male & $133(49.8)$ \\
\hline Family history of allergic diseases & $211(79.0)$ \\
\hline \multicolumn{2}{|l|}{ Indicated diseases for AIT (duplicated) } \\
\hline Asthma & $114(42.7)$ \\
\hline Allergic rhinoconjunctivitis & $244(91.4)$ \\
\hline Atopic dermatitis & $54(20.2)$ \\
\hline Previous AIT history & $27(10.2)$ \\
\hline Mean duration of AIT (month) & $27.3 \pm 15.0(12-90)$ \\
\hline \multicolumn{2}{|l|}{ Information source of AIT } \\
\hline General hospital physician & $165(69.6)$ \\
\hline General practitioner & $14(5.9)$ \\
\hline Acquaintances & $33(13.9)$ \\
\hline Internet/media & $19(8.0)$ \\
\hline Other patients & $1(0.4)$ \\
\hline Others & $5(2.1)$ \\
\hline \multicolumn{2}{|l|}{ Subjective adherence to AIT (patients) } \\
\hline Excellent & $239(91.6)$ \\
\hline Good & $7(2.7)$ \\
\hline Pair & $8(3.1)$ \\
\hline Poor & $4(1.5)$ \\
\hline Very poor & $3(1.1)$ \\
\hline
\end{tabular}

AIT, allergen-specific immunotherapy.

Family history represents to have allergic diseases in the direct blood relatives of the patient. Data are presented as a mean \pm SD (range) or number (percentage) dramatically decreased after AIT for asthma (intermittent from $7.9 \%$ to $63.4 \%$, mild persistent from $38.6 \%$ to $28.7 \%$, moderate persistent from $46.5 \%$ to from $6.9 \%$, and severe persistent $6.9 \%$ to $1.0 \%, p<0.001$ ) (Fig. 1C).

For patients with rhinitis, there were improvements in symptoms, including rhinorrhea (92.3\%), nasal obstruction (87.2\%), sneezing (88.0\%), itching (82.2\%), eye itching (80.1\%), and eye redness (76.1\%) (Fig. 1B), as well as disease severity (mild intermittent from $0 \%$ to $64.8 \%$, moderate-severe intermittent from $8 \%$ to $20.2 \%$, mild persistent from $10.8 \%$ to $14.1 \%$, and moderate-severe persistent from $81.2 \%$ to $0.9 \%$; $p<0.001$ ), (Fig. 1D).

Interestingly, about $15 \%$ of patients reported symptom improvements within 3 months after starting AIT, and almost 3 out of 4 patients reported that their symptoms were diminished within 1 year after starting AIT (Fig. 2). However, whether patients had only one of rhinitis or asthma or both diseases did not affect satisfaction with AIT among patients and their physicians.

\section{Changes in medication use after AIT}

Prescription rates were evaluated for individual drugs in the questionnaire for physicians. Before starting AIT, ICS, betaagonists, LTRA, and theophylline were administered to 79.8, $61.1,57.5$, and $2.9 \%$ of the asthmatic patients, respectively; however, the prescription rates dramatically dropped to 49.1, 37.5, 29.7, and 1\%, respectively, after AIT treatment (Fig. 3A).

Drug prescription rates were also investigated in patients with allergic rhinitis. The prescription rates of intranasal corticosteroid, antihistamines, and LTRA were 82.5 , 89.8, and $66.9 \%$, respectively, before starting AIT. The prescription rates were significantly decreased to $35.6,48.8$, and $32.8 \%$, respectively, after AIT (Fig. 3B).

\section{Medical and socioeconomic aspects of AIT}

As for advantages of AIT, disease aggravation and drug demand were decreased such that patients reported a better than 'good' state in $84.3 \%$ and $83.0 \%$ of patients, respectively, and $79.2 \%$ of patients were in a better than 'good' state with respect to adverse reactions to AIT. Regarding economic aspects of AIT, $78.7 \%$ and $83.2 \%$ of patients reported a better than 'good' state for time consumption with AIT treatment and methodologic discomfort of AIT; however, $53.6 \%$ of patients described burden with the cost of AIT (Fig. 4). In addition, the rates of overall satisfaction with AIT higher than 'good' were $85.3 \%$ and $86.4 \%$, respectively, in patients with asthma and allergic rhinitis (Fig. 5), and these were corresponded with those results of their physicians (kappa agreement $=0.234, p<0.001$ in allergic rhinitis and kappa agreement $=0.373, p<0.001$ in asthma). Interestingly, the rates of satisfaction with AIT for asthma tended to be higher in patients than in physicians.

Moreover, $91 \%$ of patients wanted to continue AIT, and 


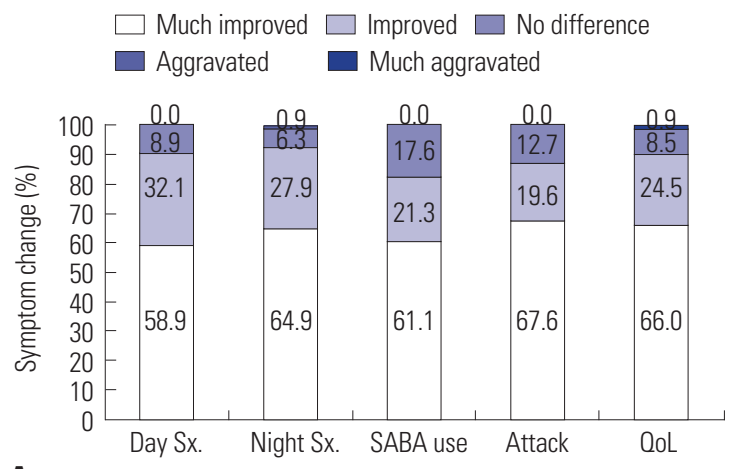

A

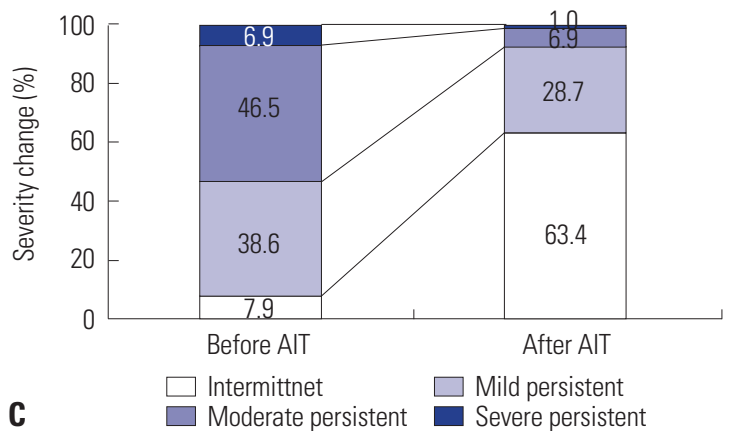

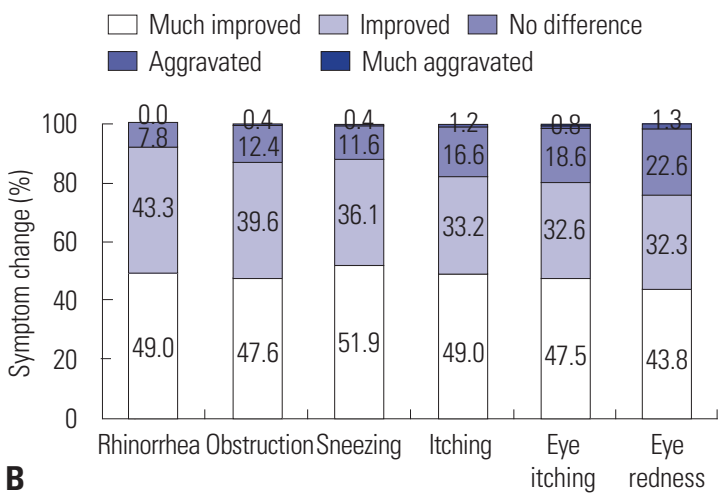

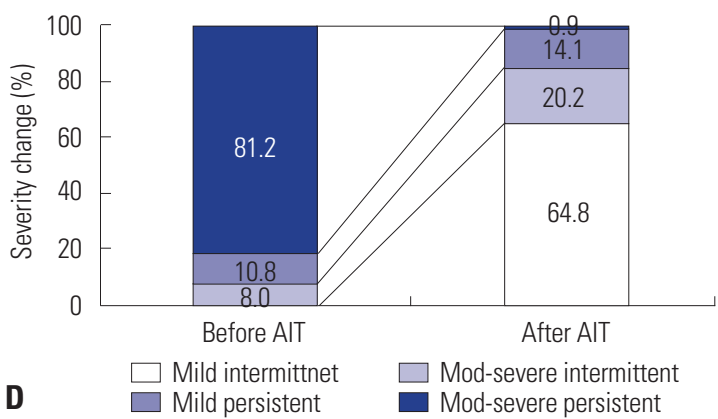

Fig. 1. Changes in allergy symptom and severity after AIT. (A) Symptom changes in patients with asthma. (B) Symptom changes in patients with allergic rhinitis. (C) Asthma severity change based on the GINA guideline. (D) Allergic rhinitis severity change based on the ARIA guideline. AIT, allergen-specific immunotherapy; SABA, short-acting bata-agonist; QoL, quality of life.

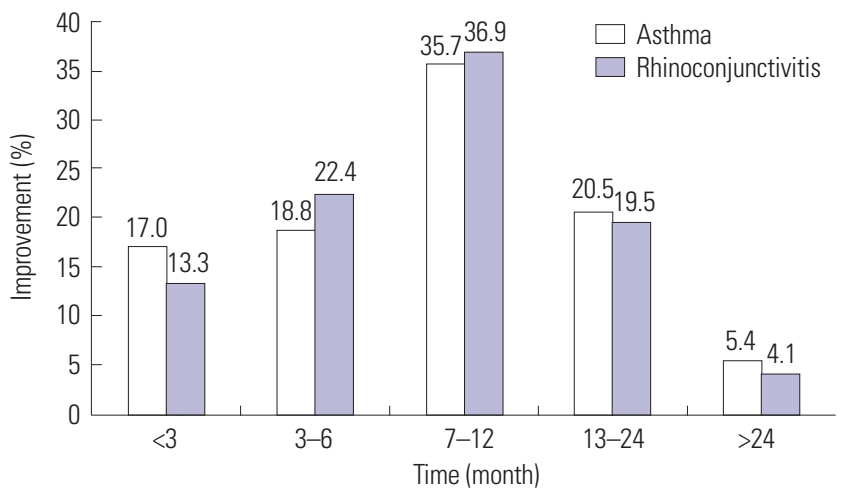

Fig. 2. Time to symptom improvement after allergen-specific immunotherapy.

88.7\% were willing to recommend AIT for other allergic patients. The results demonstrated that AIT can be a safe and effective therapeutic option for allergic patients in both medical and economic aspects, except for its cost.

\section{The efficacy of AIT according to age groups}

The efficacy of AIT was assessed in the pediatric and adult groups. Although parameters of allergic rhinitis/asthma symptoms or adverse reactions were not different between the groups (data not shown), there was a significant difference in guideline-based disease severity and medication use. The proportions of patients with intermittent severity asthma were $86.2 \%$ and $54.2 \%$, respectively, in the pediatric and adult groups after AIT. The use of inhaled corticosteroids (ICS) ( $28.1 \%$ vs. $57.7 \%$ ), beta-agonists ( $22.6 \%$ vs. $44.6 \%$ ), and leukotriene receptor antagonists (LTRA) (9.4\% vs. $36.8 \%$ ) were significantly less frequent in the pediatric group than in the adult group. Moreover, the prescription rates of antihistamine were quite decreased in pediatric allergic rhinitis patients compared to those in adult patients (Table 2).

The overall rates of satisfaction with AIT for asthma were significantly higher in the pediatric group than in the adult group ( $1.83 \pm 0.66$ vs. $1.64 \pm 0.77, p=0.040)$. However, such results were not observed in allergic rhinitis patients who received AIT (Table 2). Sex, adherence to AIT, initial disease severities, and associated diseases did not affect satisfaction with AIT in both asthma and rhinitis patients.

Taken together, our results showed that AIT might reduce allergy symptoms, medication use and disease severity based on the GINA or ARIA guidelines in Korean patients with asthma or allergic rhinitis.

\section{DISCUSSION}

In this cross-sectional study, we aimed to evaluate patient perspectives on AIT together with those of their physicians, and found excellent satisfactions with AIT in regarding to its efficacy and time-consuming and methodologic difficulties in both groups with good correlation. We also clearly showed 

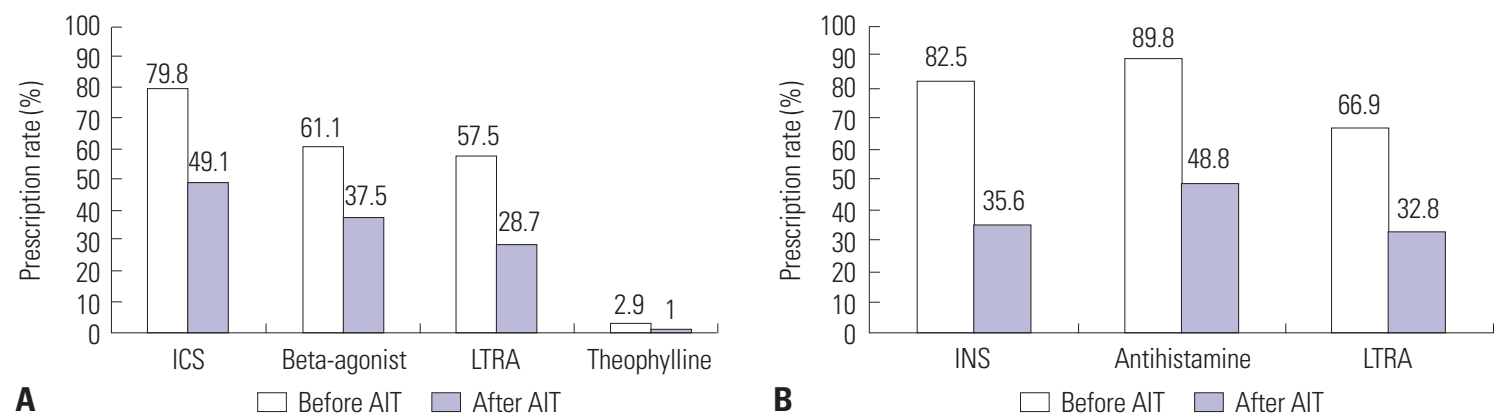

Fig. 3. Changes in drug prescription patterns after AIT. (A) Prescription patterns after AIT in patients with asthma. (B) Prescription patterns after AIT in patients with allergic rhinitis. AIT, allergen-specific immunotherapy; ICS, inhaled corticosteroids; LTRA, leukotriene receptor antagonists; INS, intranasal corticosteroid.
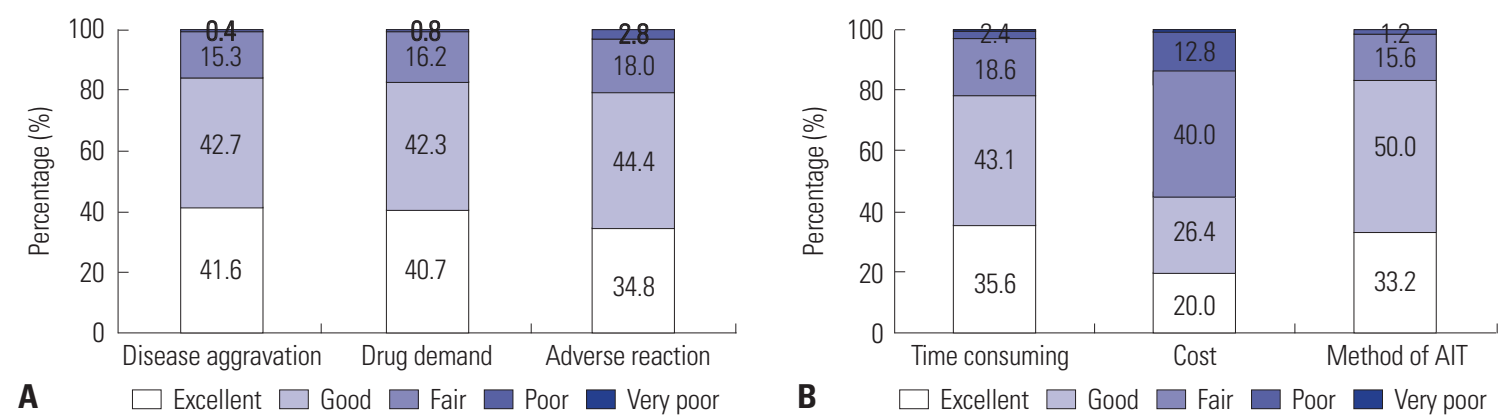

Fig. 4. The subjective advantage of AIT in medical (A) and economic and methodologic aspects (B) from the patients' perspective. AIT, allergen-specific immunotherapy.
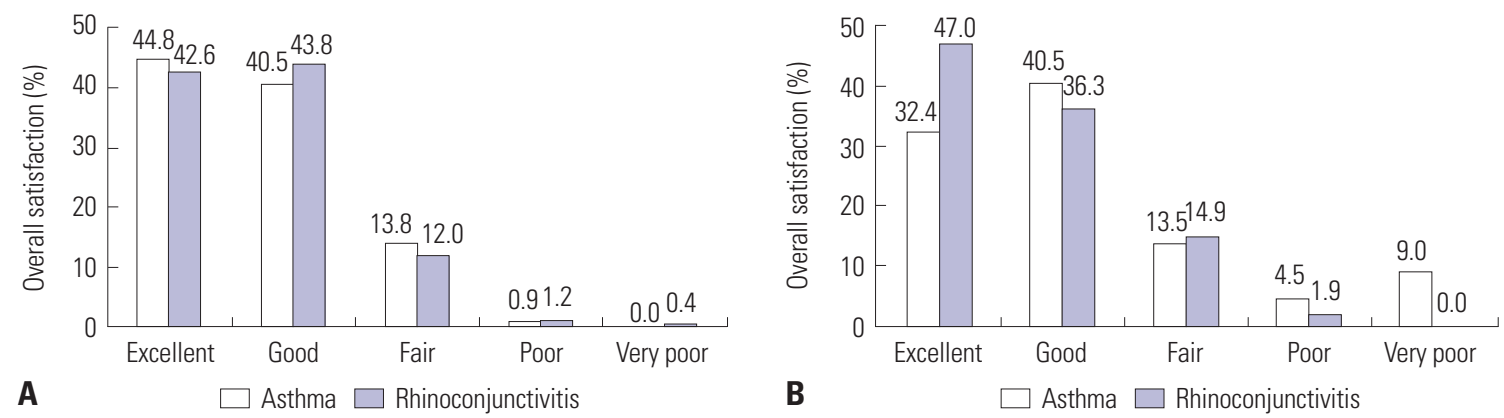

Fig. 5. Overall satisfaction of allergen-specific immunotherapy in patients $(A)$ and physicians (B).

that about three-quarter of the patients experienced symptom improvement within a year after AIT in this study.

Although many surveys of prescription patterns in AIT have been published, ${ }^{23,24}$ studies regarding perspectives or satisfaction with AIT are extremely rare, especially in Korea. This study had some heterogeneity in disease patterns, hospital settings, ages, and products for AIT and the specialties of physicians due to its retrospective design. Despite this heterogeneity, it is interesting that the overall rate of satisfaction with AIT was more than $80 \%$ for asthma or allergic rhinitis patients, moreover, it was more than $70 \%$ for physicians. This result is in line with those of previous studies performed in Italy and Spain. ${ }^{19,20}$ The rates of patient and physician satisfaction with AIT were 74/100 and 78/100, respectively, using VAS scores, and showed a significant similarity between them. ${ }^{25}$ The Satis- faction Scale for Patients Receiving Allergen Immunotherapy (ESPIA) totaled 69.2 points on a 0-100 scale after sublingual grass pollen immunotherapy. ${ }^{26}$ Although the methods for evaluating the overall rate of satisfaction with AIT were not similar between studies, AIT appeared to be an excellent therapeutic option meeting the expectations of allergic patients and physicians. In contrast, the most unsatisfactory part had answered the cost of AIT. More than $50 \%$ of patients answered fair or worse opinions in that question.

Another interesting result of this study was that the rate of satisfaction with AIT was higher in pediatric asthmatics than in adult asthmatics. While patient age does not seem to be a limitation to AIT, the efficacy of AIT according to age groups is still debatable. Asthma is a chronic inflammatory disease that leads to irreversible changes in the airway. Therefore, it would 
Table 2. Effects of AIT according to Age Groups

\begin{tabular}{|c|c|c|c|c|}
\hline & Age $\leq 15$ & Age $>15$ & Total & $p$ value \\
\hline Asthma severity after AlT & & & & 0.004 \\
\hline Intermittent & $25(86.2)$ & 39 (54.2) & $64(63.4)$ & \\
\hline Mild persistent & $3(10.3)$ & $26(36.1)$ & $29(28.7)$ & \\
\hline Moderate persistent & $0(0)$ & $7(9.7)$ & $7(6.9)$ & \\
\hline Severe persistent & $1(3.4)$ & $0(0)$ & $1(1)$ & \\
\hline Rhinitis severity after AIT & & & & 0.794 \\
\hline Mild intermittent & 38 (67.9) & $100(63.7)$ & $138(64.8)$ & \\
\hline Mod-severe intermittent & $10(17.9)$ & $33(21.0)$ & $43(20.2)$ & \\
\hline Mild persistent & $8(14.3)$ & $22(14.0)$ & $30(14.1)$ & \\
\hline Mod-severe persistent & $0(0)$ & $2(1.3)$ & $2(0.9)$ & \\
\hline Beta-agonist use after AIT & $7(22.6)$ & $29(44.6)$ & $36(37.5)$ & 0.044 \\
\hline ICS use after AIT & $9(28.1)$ & $45(57.7)$ & $54(49.1)$ & 0.006 \\
\hline LTRA use after AIT (asthma) & $3(9.4)$ & $28(36.8)$ & $31(28.7)$ & 0.005 \\
\hline INS use after AIT & $20(42.6)$ & $48(33.3)$ & $68(35.6)$ & 0.293 \\
\hline Antihistamine use after AIT & $19(34.5)$ & $82(53.9)$ & $101(48.8)$ & 0.018 \\
\hline LTRA use after AIT (rhinitis) & $17(30.4)$ & 40 (33.9) & 57 (32.8) & 0.730 \\
\hline Overall satisfaction in asthma & $1.83 \pm 0.66$ & $1.64 \pm 0.77$ & $1.71 \pm 0.73$ & 0.040 \\
\hline Overall satisfaction in rhinitis & $2.99 \pm 1.08$ & $2.85 \pm 1.27$ & $2.90 \pm 1.20$ & 0.736 \\
\hline
\end{tabular}

AIT, allergen-specific immunotherapy; ICS, inhaled corticosteroids; LTRA, leukotriene receptor antagonists; INS, intranasal corticosteroid.

Data are presented as a number (percentage) or mean \pm SD.

be advisable to start AIT earlier in the disease process. Our data showed no differences in allergy symptoms between patients of ages less than 15 years and those older than 15 years, but showed a significant difference in guideline-based disease severity and medication use after AIT, especially in asthmatics. Previous studies have reported similar results as ours. SLIT with house dust mite extracts seems to be as effective in school-age children and adolescents as in adults; however, the degree of reduction in medication use tended to be higher in the young age group than in the adolescent group. ${ }^{27}$ Interestingly, a significant difference in disease severity was found only in asthma, but not in allergic rhinitis. Airway remodeling such as collagen deposition could prominently occur in suboptimally treated or untreated asthma, in contrast, those features are not prominent in allergic rhinitis. We thought the above difference may attribute to our results in asthma and rhinitis. Moreover, some discrepancies in satisfaction rates with AIT for asthma between patients and physicians may have been attributed to the lack of objective monitoring tools such as pulmonary function tests to patients. Further studies are needed to confirm our findings.

Patients should be educated about AIT before the start of AIT because AIT requires a long treatment duration and sometimes causes hypersensitivity reactions. In this study, only $5.9 \%$ of the patients had heard about AIT from general practitioners. The Internet, media, or acquaintances were important sources of information on AIT. This could disseminate incorrect knowledge about AIT to many allergic patients. This finding was also in line with those of previous studies con- cerning the sources of information on AIT. ${ }^{13,15,25}$ Approximately $30 \%$ of patients expected immediate therapeutic effects from AIT, and about $20 \%$ of the patients wanted the treatment to be performed for a short period of time. ${ }^{25}$ Our results can explain the reasons why AIT is not frequently offered to patients and why many patients hold misconceptions about AIT in Korea. We should consider the education of general practitioners and the use of multimedia for better understanding and more prescriptions of AIT in Korea.

AIT significantly decreased all clinical symptoms in patients with asthma or allergic rhinitis as reported in previous studies. ${ }^{28,29}$ More than $80 \%$ of patients reported an 'improved' or 'much improved' state in regarding to clinical symptoms after AIT. In addition, disease severity, an objective parameter, was also reduced after AIT in many patients. In this study, $81.2 \%$ of allergic rhinitis patients showed a moderate to severe persistent state before AIT; $64.8 \%$ of the patients changed to mild intermittent state; and only $20.2 \%$ of the patients showed a moderate to severe intermittent state after AIT. Similarly, $7.9 \%$ of intermittent asthma patients turned to $63.4 \%$ after AIT. These results are also consistent with those of previous studies demonstrating that the severity of allergic rhinitis was reduced from moderate to severe severity to mild severity in $49.1 \%$ of patients. ${ }^{26}$ This study also showed the decreased medication prescription rate after AIT in both allergic rhinitis and asthma patients. Taken together, AIT might be very effective in improving disease severity and reducing the frequency of medication use.

Our survey evaluated the time of symptom improvement. About $15 \%$ of patients reported symptom improvement within 3 months, more than $30 \%$ of the patients within 6 months, and $75 \%$ of patients within 1 year. It is conceivable that AIT can show quick therapeutic effects through early desensitization of mast cells within 1 week and production of regulatory cytokines, such as IL-10 and TGF-beta, within 6 months. ${ }^{30,31}$ Although further studies are needed to confirm our findings, time to symptom improvement can help to explain the onset timing of AIT to allergic patients.

Our study has some limitations. First, we only analyzed results from patients receiving subcutaneous immunotherapy. In a previous study in Korea, SLIT was usually prescribed by otolaryngologists. ${ }^{32}$ However, enrolled doctors in our study were only from internal medicine and pediatrics. This is the reason why the number of patients receiving SLIT was small in our study. Secondly, we could not compare the efficacy and safety of AIT between AIT products or administration methods. The questionnaire developed by an expert panel of the allergen and immunotherapy workgroup of the KAAACI was inadequate for these purposes. Our workgroup is planning to compare AIT products in future studies. However, the strengths of the present study are that data were collected in real-life settings and that relevant data based on current guidelines were provided for the management of patients re- 
ceiving AIT, emphasizing the importance of the patient's point of view on AIT. Thirdly, since some children may have spontaneous remissions of allergic symptoms as they become older, we cannot confirm the superiority of AIT in childhood asthma on the basis of the above results.

In conclusion, in this study evaluating the perspectives on AIT in both patients and physicians, the majority of patients and physicians were satisfied with the efficacy and safety of AIT, except for its cost. Physicians who treat allergic patients should recognize the strength and weakness of AIT and recommend more AIT to allergic patients.

\section{ACKNOWLEDGEMENTS}

This work was supported by a grant from the Korean Academy of Asthma, Allergy and Clinical Immunology (KAAACI).

\section{AUTHOR CONTRIBUTIONS}

Conceptualization: Yoo Seob Shin and Jae-Woo Jung. Data curation: Jae-Woo Kwon and Sang Min Lee. Formal analysis: Yoo Seob Shin and Ji Hye Kim. Funding acquisition: Sooyoung Lee and Jung-Won Park. Investigation: Yoo Seob Shin, Jae-Woo Jung, and Jeong-Hee Choi. Methodology: Jae-Woo Jung, Jae-Woo Kwon, and Jung-Won Park. Project administration: Yoo Seob Shin. Resources: Ji Hye Kim, Sang Min Lee, and Jae-Woo Kwon. Software: Yoo Seob Shin, Jae-Woo Jung, and Sooyoung Lee. Supervision: Man Yong Han, Young Min Ahn, and Jung-Won Park. Validation: Jeong-Hee Choi and Jae-Woo Jung. Visualization: Sooyoung Lee and Sang Min Lee. Writing-original draft: Yoo Seob Shin and Jeong-Hee Choi. Writing-review \& editing: Man Yong Han and Young Min Ahn.

\section{ORCID iDs}

Yoo Seob Shin Jae-Woo Jung Jung-Won Park Jeong-Hee Choi Jae-Woo Kwon Sooyoung Lee Ji Hye Kim Sang Min Lee Young Min Ahn Man Yong Han https://orcid.org/0000-0002-9855-3185 https://orcid.org/0000-0002-3411-735X https://orcid.org/0000-0003-0249-8749 https://orcid.org/0000-0002-0599-875X https://orcid.org/0000-0003-1639-3606 https://orcid.org/0000-0003-1734-4101 https://orcid.org/0000-0003-4677-0513 https://orcid.org/0000-0002-9568-2096 https://orcid.org/0000-0002-1697-8041 https://orcid.org/0000-0002-9077-5779

\section{REFERENCES}

1. Noon L. Prophylactic inoculation against hay fever. Int Arch Allergy Appl Immunol 1953;4:285-8.

2. Varney VA, Tabbah K, Mavroleon G, Frew AJ. Usefulness of specific immunotherapy in patients with severe perennial allergic rhinitis induced by house dust mite: a double-blind, randomized, placebo-controlled trial. Clin Exp Allergy 2003;33:1076-82.

3. Blumberga G, Groes L, Haugaard L, Dahl R. Steroid-sparing effect of subcutaneous SQ-standardised specific immunotherapy in moderate and severe house dust mite allergic asthmatics. Allergy 2006;61:843-8.

4. Niggemann B, Jacobsen L, Dreborg S, Ferdousi HA, Halken S, Høst
A, et al. Five-year follow-up on the PAT study: specific immunotherapy and long-term prevention of asthma in children. Allergy 2006;61:855-9.

5. Durham SR, Emminger W, Kapp A, de Monchy JG, Rak S, Scadding GK, et al. SQ-standardized sublingual grass immunotherapy: confirmation of disease modification 2 years after 3 years of treatment in a randomized trial. J Allergy Clin Immunol 2012;129:717-25.

6. Jacobsen L, Niggemann B, Dreborg S, Ferdousi HA, Halken S, Høst A, et al. Specific immunotherapy has long-term preventive effect of seasonal and perennial asthma: 10-year follow-up on the PAT study. Allergy 2007;62:943-8.

7. Novembre E, Galli E, Landi F, Caffarelli C, Pifferi M, De Marco E, et al. Coseasonal sublingual immunotherapy reduces the development of asthma in children with allergic rhinoconjunctivitis. J Allergy Clin Immunol 2004;114:851-7.

8. Inal A, Altintas DU, Yilmaz M, Karakoc GB, Kendirli SG, Sertdemir Y. Prevention of new sensitizations by specific immunotherapy in children with rhinitis and/or asthma monosensitized to house dust mite. J Investig Allergol Clin Immunol 2007;17:85-91.

9. Reha CM, Ebru A. Specific immunotherapy is effective in the prevention of new sensitivities. Allergol Immunopathol (Madr) 2007; 35:44-51.

10. Hankin CS, Cox L, Lang D, Levin A, Gross G, Eavy G, et al. Allergy immunotherapy among Medicaid-enrolled children with allergic rhinitis: patterns of care, resource use, and costs. J Allergy Clin Immunol 2008;121:227-32.

11. Law AW, Reed SD, Sundy JS, Schulman KA. Direct costs of allergic rhinitis in the United States: estimates from the 1996 Medical Expenditure Panel Survey. J Allergy Clin Immunol 2003;111:296-300.

12. Anolik R, Schwartz AM, Sajjan S, Allen-Ramey F. Patient initiation and persistence with allergen immunotherapy. Ann Allergy Asthma Immunol 2014;113:101-7.

13. Demoly P, Didier A, Mathelier-Fusade P, Drouet M, David M, Bonnelye G, et al. Physician and patient survey of allergic rhinitis in France: perceptions on prevalence, severity of symptoms, care management and specific immunotherapy. Allergy 2008;63:1008-14.

14. Lombardi C, Canonica GW, Passalacqua G. The perception of allergen-specific immunotherapy among chest physicians: an Italian survey. Eur Ann Allergy Clin Immunol 2014;46:132-6.

15. Lombardi C, Bettoncelli G, Canonica GW, Passalacqua G. The perception of allergen-specific immunotherapy among Italian general practitioners. Eur Ann Allergy Clin Immunol 2012;44:80-2.

16. Radulovic S, Calderon MA, Wilson D, Durham S. Sublingual immunotherapy for allergic rhinitis. Cochrane Database Syst Rev 2010; 12:CD002893.

17. Bożek A, Kołodziejczyk K, Kozłowska R, Canonica GW. Evidence of the efficacy and safety of house dust mite subcutaneous immunotherapy in elderly allergic rhinitis patients: a randomized, double-blind placebo-controlled trial. Clin Transl Allergy 2017;7:43.

18. Virchow JC, Backer V, Kuna P, Prieto L, Nolte H, Villesen HH, et al. Efficacy of a house dust mite sublingual allergen immunotherapy tablet in adults with allergic asthma: a randomized clinical trial. JAMA 2016;315:1715-25.

19. Serrano P, Justicia JL, Sánchez C, Cimarra M, Fernández-Távora L, Orovitg A, et al. Systemic tolerability of specific subcutaneous immunotherapy with index-of-reactivity-standardized allergen extracts administered using clustered regimens: a retrospective, observational, multicenter study. Ann Allergy Asthma Immunol 2009; 102:247-52.

20. Ciprandi G, Cadario G, Valle C, Ridolo E, Verini M, Di Gioacchino $\mathrm{M}$, et al. Sublingual immunotherapy in polysensitized patients: effect on quality of life. J Investig Allergol Clin Immunol 2010;20: 274-9. 
21. Wyrzykowska N, Czarnecka-Operacz M, Adamski Z. Long-term efficacy of allergen specific immunotherapy in atopic dermatitis patients in relation to quality of life. Eur Ann Allergy Clin Immunol 2015;47:5-9.

22. Rak S, Yang WH, Pedersen MR, Durham SR. Once-daily sublingual allergen-specific immunotherapy improves quality of life in patients with grass pollen-induced allergic rhinoconjunctivitis: a double-blind, randomised study. Qual Life Res 2006;16:191-201.

23. Lombardi C, Senna G, Passalacqua G. Specific immunotherapy among Italian specialists. Allergy 2006;61:898-9.

24. Coifman RE, Cox LS; Immunotherapy and Allergy Diagnostics Committee of the AAAAI. 2006 American Academy of Allergy, Asthma \& Immunology member immunotherapy practice patterns and concerns. J Allergy Clin Immunol 2007;119:1012-3.

25. Baiardini I, Puggioni F, Menoni S, Boot JD, Diamant Z, Braido F, et al. Patient knowledge, perceptions, expectations and satisfaction on allergen-specific immunotherapy: a survey. Respir Med 2013; 107:361-7.

26. Antolín-Amerigo D, Tabar IA, Del Mar Fernández-Nieto M, Callejo-Melgosa AM, Muñoz-Bellido FJ, Martínez-Alonso JC, et al. Satisfaction and quality of life of allergic patients following sublingual five-grass pollen tablet immunotherapy in Spain. Drugs Context 2017;6:212309.

27. Han DH, Choi YS, Lee JE, Kim DY, Kim JW, Lee CH, et al. Clinical efficacy of sublingual immunotherapy in pediatric patients with al- lergic rhinitis sensitized to house dust mites: comparison to adult patients. Acta Otolaryngol 2012;132 Suppl 1:S88-93.

28. Frew AJ, Ljørring C, Wolf H, Wüstenberg E, Durham SR, Corrigan CJ, et al. UK Immunotherapy study: reanalysis by a combined symptom and medication score. J Allergy Clin Immunol 2018;142: 1998-9.

29. Grouin JM, Vicaut E, Devillier P. Comparison of scores associating symptoms and rescue medication use for evaluating the efficacy of allergy immunotherapy in seasonal allergic rhinoconjunctivitis: results from five trials. Clin Exp Allergy 2017;47:254-63.

30. Burks AW, Calderon MA, Casale T, Cox L, Demoly P, Jutel M, et al. Update on allergy immunotherapy: American Academy of Allergy, Asthma \& Immunology/European Academy of Allergy and Clinical Immunology/PRACTALL consensus report. J Allergy Clin Immunol 2013;131:1288-96.

31. Tordesillas L, Mondoulet L, Blazquez AB, Benhamou PH, Sampson HA, Berin MC. Epicutaneous immunotherapy induces gastrointestinal LAP+ regulatory T cells and prevents food-induced anaphylaxis. J Allergy Clin Immunol 2017;139:189-201.

32. Hur GY, Kim TB, Han MY, Nahm DH, Park JW; Allergen and Immunotherapy Work Group of the Korean Academy of Asthma, Allergy and Clinical Immunology (KAAACI). A survey of the prescription patterns of allergen immunotherapy in Korea. Allergy Asthma Immunol Res 2013;5:277-82. 